
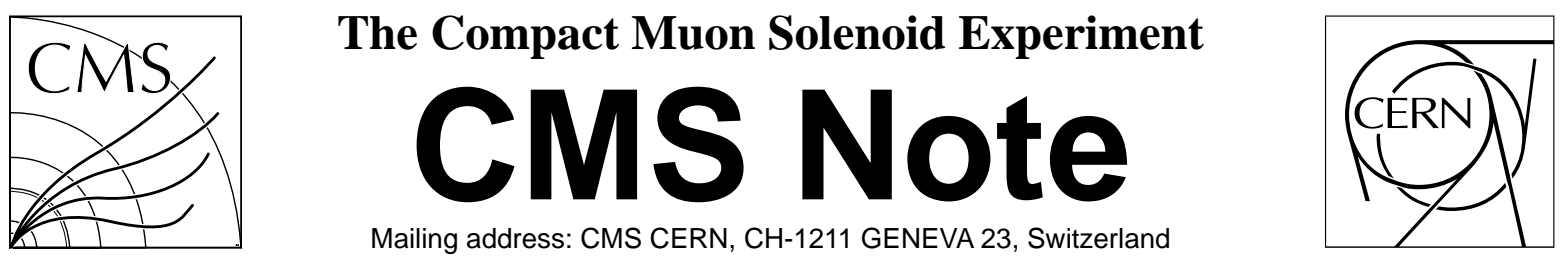

25 July 2001

\title{
Performance of a CMS silicon strip detector module with APV25 readout
}

\author{
M. Friedl*), T. Bauer, J. Hrubec, M. Krammer, M. Pernicka \\ Institute of High Energy Physics, Austrian Academy of Sciences, Vienna, Austria
}

\begin{abstract}
The Compact Muon Solenoid experiment (CMS) at the Large Hadron Collider (LHC) at CERN will include a Silicon Strip Tracker covering a sensitive area of $206 \mathrm{~m}^{2}$ with about ten million readout channels. Its silicon detectors, made from 6" wafers, will be read out by APV25 front-end chips, fabricated in the $0.25 \mu \mathrm{m}$ deep submicron process which is intrinsically radiation-tolerant. A first prototype module has been built consisting of two chained silicon sensors of $320 \mu \mathrm{m}$ thickness and three APV25 chips (version S0). The performance of this module has been evaluated in a pion and proton beam at the Paul Scherrer Institute (Villigen/CH).
\end{abstract}

Key words: APV25, Silicon Detector, Module, Tracker, CMS, LHC, CERN

Submitted to Nuclear Instruments and Methods A

*) Corresponding Author, Email: Markus.Friedl@cern.ch 


\section{Introduction}

In late 1999, the CMS collaboration decided to change the design of the tracker, which was previously based on silicon strip detectors and Micro-Strip Gas Chambers, and to construct a Silicon Strip Tracker with a total area of $206 \mathrm{~m}^{2}[1,2]$. The silicon sensors will be made from 6" wafers, resulting in a maximum strip length of $21 \mathrm{~cm}$ by chaining two sensors. In addition to the $320 \mu \mathrm{m}$ thick detectors at smaller radii, sensors with $500 \mu \mathrm{m}$ thickness will be used to compensate the additional noise of the long detectors in the outer part of the tracker by a higher energy deposition. The strip pitches vary between 61 and $205 \mu \mathrm{m}$.

The high particle rates at full LHC operation and the resulting radiation levels in the tracker volume require the use of radiation-hard front-end electronics. The APV25 chip [3], designed to read out the CMS silicon strip detectors, is therefore manufactured in the industry-standard $0.25 \mu \mathrm{m}$ technology, which is an economic alternative to expensive specialized radiation-tolerant processes by providing an intrinsic radiation hardness due to its thin gate oxides. The APV25 is a redesign of the APV6 prototype [4] with significantly improved noise characteristics and including additional features.

The APV25 chip will be driven synchronously by the LHC clock at $40 \mathrm{MHz}$. Each chip contains 128 channels with a preamplifier, a CR-RC shaper with a time constant of $50 \mathrm{~ns}$ and a 192-cell analog pipeline. The implemented deconvolution algorithm [5] narrows the pulses and thus allows to identify precisely the LHC bunch crossing from which a signal originates. After receiving a trigger signal, the analog data are multiplexed to the serial output line. Alternatively, the APV25 can be operated in peak mode without deconvolution or in a multi-peak mode, where for each trigger the signals of three consecutive time slots are presented at the output.

\section{Experimental Setup}

Before producing strip detectors in larger quantities for CMS it was of great interest to measure the characteristic parameters of a detector with realistic dimensions for minimum ionizing particles and at particle rates comparable to the future LHC operation.

The first silicon detector module with an APV25 readout (fig. 1) was built at our institute in early 2000. It consisted of two sensors, made from 6" wafers of $320 \mu$ m thickness, with a total strip length of $16.5 \mathrm{~cm}$ and a strip pitch of $140 \mu \mathrm{m}$. The high silicon bulk resistivity resulted in a low depletion voltage of approximately $70 \mathrm{~V}$. The detector module was partially read out by three APV25 chips (version S0), which were mounted on a dedicated printed circuit board (PCB) hybrid and connected to the sensors through a two-stage pitch adapter.

This APV25 detector module was tested at the Paul Scherrer Institute in a $350 \mathrm{MeV} / \mathrm{c}$ particle beam, delivering pions (which are almost minimum ionizing particles) and protons. For most of the measurements, the protons were removed by an aluminum shield in front of the bending magnets of the beamline. A scintillator counter $\left(12 \times 12 \mathrm{~mm}^{2}\right)$ behind the module was used to trigger on particles traversing approximately the center of the module. The module was operated in a cooling box at $-10^{\circ} \mathrm{C}$ to provide conditions similar to CMS. As an exception, for the angle scan the module had to be run at ambient temperature due to mechanical constraints.

The APV25 chips were driven by the nominal LHC bunch crossing frequency of $40 \mathrm{MHz}$. A custom Phase Locked Loop circuit was developed to derive the APV clock from the $50 \mathrm{MHz}$ PSI beam timing together with a synchronization pulse to select scintillator triggers which are in phase with both clocks. Most of the data have been taken at incident particle rates of about $10 \mathrm{kHz} \mathrm{cm}^{-2}$. For a $24 \mathrm{~h}$ period of high intensity the beam was tuned to deliver particles at $1.4 \mathrm{MHz} \mathrm{cm}^{-2}$, exceeding the expected particle rate at the innermost radius of the CMS Silicon Strip Tracker during LHC operation.

At our institute, a complete VME-based APV readout system [6] has been developed, including an APV sequencer, $\mathrm{I}^{2} \mathrm{C}$ bus masters, 12-bit ADCs at $40 \mathrm{MHz}$ and supporting hardware. Fig. 2 shows a schematic diagram of this setup. A comprehensive PC data acquisition software with online analysis communicated with the electronics. A second PC was used for slow controls and monitoring purposes, controlling the cooling system and the detector bias. An extended version of the data acquisition software was used for offline analysis. In every run, 600 random-triggered initial events were used for pedestal and noise evaluation, while the following events (typically 40k) were subjected to zero-suppression and clustering algorithms. All data were corrected for common-mode noise. For our analysis, we used cluster cuts of $6 \sigma$ for the central seed strip and $3 \sigma$ for the neighboring strips in terms of the strip RMS noise. 


\section{Results}

\subsection{Signal Distribution}

In fig. 3, the distributions of cluster signals are shown for $350 \mathrm{MeV} / \mathrm{c}$ pions and protons. To fit the measured signal distribution of the pions, a Landau function was convoluted with a narrow Gauss curve to account for electronic noise and intrinsic detector fluctuations. The distribution of the much higher signals of the protons can be almost completely described by a Gauss function. The measured signals match very well with calculations of the restricted energy loss in silicon in the framework of the Bethe-Bloch theory. A calculated ratio of 6.0 between the mean energy loss of protons and pions compares to a measured quotient of 6.6.

\subsection{Signal-to-Noise Ratio}

The signal-to-noise ratio is an important parameter for the detector efficiency. For the CMS Silicon Strip Tracker modules, a most probable signal-to-noise (SNR) greater than 10 is demanded throughout their lifetime for minimum ionizing particles, which ensures an efficiency close to $100 \%$. Fig. 4 shows the most probable SNR obtained with the APV25 detector module in the pion beam as a function of the bias voltage for both peak and deconvolution modes. The equivalent noise charge (ENC) of the detector and amplifier system can be approximately derived by dividing the charge deposited by a minimum ionizing particle by the signal-to-noise ratio. Tab. 1 compares the SNR and ENC values obtained with the APV25 detector module at full depletion to similar measurements performed with an APV6 readout [6].

The detector strip length determines the load capacitance seen by the amplifier and thus influences the electronic noise and the SNR. Moreover, the collected charge signal decreases with increasing irradiation due to the creation of crystal defects which act as charge traps. In addition, the detector noise increases with the rising leakage current, both effects leading to a SNR reduction. Nevertheless, the APV25 performance offers sufficient margin to maintain excellent efficiency even after 10 years of LHC operation.

\subsection{Module Robustness}

To check for a possible signal degradation along the strips, the scintillator behind the APV25 module was moved to several positions along the strip direction, by that defining different areas under test. As expected from the electrical properties of signal generation, no difference in the performance was obtained with respect to the position along the strip axis. As a particle crossing the detector represents a current source, the integral charge (which is measured by the amplifier) is not affected by varying the strip series resistance.

Driven by the APV clock at $40 \mathrm{MHz}$, sampled values of the APV25 shaper are stored for each readout channel in the chip-internal analog pipeline and retrieved up to 192 clock cycles (corresponding to $4.8 \mu \mathrm{s}$ ) later. By variation of this latency time, neither signal nor noise were influenced, demonstrating the ability of the APV25 pipeline to hold the signal without noticeable leakage beyond the required CMS first-level trigger latency.

\subsection{Optical Link}

The APV25 output signals were usually transmitted through a copper cable with a length of $25 \mathrm{~m}$, but alternatively, a prototype of the CMS analog optical link [7] with $100 \mathrm{~m}$ of single-mode fiber, was installed for comparative measurements. Contrary to the expectation of a reduced signal-to-noise ratio due to the additional noise contribution of the link, an approximately $7 \%$ higher SNR was measured. This was because the long copper cable caused a bandwidth limitation which led to a slight reduction of the signal compared to the optical link which had a significantly higher bandwidth.

The prototype optical link used in this test with the APV25 detector module had a higher gain and therefore a lower noise contribution compared to the optical link foreseen for the final tracker readout. Nevertheless, the final analog optical link is expected to contribute less than 850 e noise [8].

\subsection{High Intensity}

During the 24-hour-period of high intensity pion beam $\left(1.4 \mathrm{MHz} \mathrm{cm}^{-2}\right)$, the detector performance remained unchanged in terms of signal and noise. However, the leakage current as shown in fig. 5 increased as a linear function of the fluence accumulated in time. Initially, the current was very low, but jumps by approximately $0.5 \mu \mathrm{A}$ as soon 
as the high intensity beam was turned on. This is caused by the large number of charge carriers generated within the detector by the crossing pions, therefore known as beam induced current.

The slope of the current in fig. 5 corresponds to the increase of the leakage current $I$ caused by radiation defects [9], which has been parameterized by

$$
\Delta I=\alpha \Phi_{\mathrm{eq}} V,
$$

where $\alpha$ is the current related damage rate, $\Phi_{\text {eq }}$ the $1 \mathrm{MeV}$ neutron equivalent fluence and $V$ the sensitive volume. Without any annealing, a current related damage rate of $\alpha \approx 8 \cdot 10^{-17} \mathrm{~A} / \mathrm{cm}$ at room temperature can be derived from this measurement, which agrees with values given by the CERN RD48 (ROSE) collaboration [9].

Occasionally, the beam went off for a few seconds, resulting in a measurement of the pure leakage current without the beam induced component. Joining these points would result in a parallel line with the same slope but at a lower level. The current spike at noon occurred due to a short power failure of the cooling system, resulting in a temporary current increase. After restoring the operating temperature, the current curve continues unaffected. Later, the beam intensity was slightly reduced for about two hours, resulting in a lower beam induced current.

\subsection{Angle Scan}

In order to study the signal properties under non-perpendicular penetration by the beam particles the APV25 detector module had to be rotated around its strip direction. Therefore these measurements were performed at room temperature outside of the cooling box. Denoting the perpendicular incidence of the beam particles with $\alpha=0^{\circ}$, the beam incidence angle $\alpha$ was varied in four steps up to $60^{\circ}$. Previous studies [10] have shown that due to the longer path of the beam particles in the detector the total collected charge increases as given by

$$
\frac{Q_{\alpha}}{Q_{0}}=\frac{1}{\cos \alpha},
$$

where $Q_{\alpha}$ is the cluster charge at the beam incidence angle $\alpha$ and $Q_{0}$ the collected charge at perpendicular incidence. Similarly, the cluster width increases as well due to geometrical reasons. The empirical function

$$
c \propto \sqrt{\tan ^{2} \alpha+p^{2}}
$$

describes the average cluster width $c$ in terms of the number of strips contributing to the cluster with the fit parameter $p$, which represents the cluster width at perpendicular incidence.

Fig. 6 shows the cluster width (top) and the signal (bottom) as functions of the beam incidence angle for both pions and protons, $\alpha=0^{\circ}$ denoting the perpendicular beam incidence. The fit functions (2) and (3) have been successfully applied. Due to the high ionization created by protons, the cluster spread is already more than 3 strips $(420 \mu \mathrm{m})$ at perpendicular incidence.

\subsection{Multi-Peak Mode}

The APV Sequencer is able to generate different programmable trigger patterns which are issued either by software or by a hardware trigger. This feature, together with the multi-peak mode of the APV25, can be used to effectively obtain subsequent samples of the shaping curve from a particle signal [6]. Fig. 7 shows an example of the APV25 output in this mode. Four triggers separated by $75 \mathrm{~ns}$ are sent to the APV25, which returns three consecutive samples after each trigger, resulting in a total of twelve sequential samples, revealing the (negative) pulse shape. As shown in fig. 8, the average waveform obtained from this measurement matches with the peak mode pulse shape scanned by internal calibration.

The multi-peak mode can be used to measure the timing stability between the APV25 clock and the particle timing. For this purpose, to each event a pulse shape fit function has been applied to determine the peaking time. Using the scintillator trigger, these peaking times have been histogrammed, resulting in a Gaussian spread with an RMS of about 2 ns. This demonstrates the high precision of the system timing, although this number still includes beam fluctuations and the fit error.

At high intensity with a random trigger (but necessarily synchronous to the 25 ns APV25 clock), similar Gaussian peaks appear regularly spaced in time, revealing the $20 \mathrm{~ns}$ structure of the beam at the Paul Scherrer Institute (fig. 9). The pulse shape fit does not always converge. With a later peak, less signal samples are contained within the measurement window, making the fit procedure more difficult. Fits which did not converge could not be included in the plot, leading to fewer entries for later peaks, although the original particle signals were evenly distributed over the full time scale. 


\section{Summary}

For the first time a CMS silicon strip detector module has been equipped with an APV25 readout. It consisted of two high resistivity sensors, produced from $320 \mu \mathrm{m}$ thick 6" wafers, and had a total strip length of $16.5 \mathrm{~cm}$. This APV 25 module was tested with $350 \mathrm{MeV} / \mathrm{c}$ pions and protons in a cooled environment at $-10^{\circ} \mathrm{C}$.

The observed signal distributions for pions and protons are in agreement with the restricted energy loss in silicon predicted by the Bethe-Bloch theory. For the $350 \mathrm{MeV} / \mathrm{c}$ pions, which are approximately minimum ionizing particles, most probable signal-to-noise ratios of 25 and 17 were obtained in peak mode and in deconvolution mode, respectively. Thus, the APV25, which is manufactured in the $0.25 \mu \mathrm{m}$ deep submicron process, outperforms its predecessor APV6 especially in terms of noise. The measured performance allows $25 \mathrm{~ns}$ bunch crossing separation with sufficient signal-to-noise margin to allow excellent efficiency even with radiation induced degradation after 10 years of LHC operation.

Moreover, a prototype of the CMS analog optical link was successfully tested in the module readout path. During a high-intensity beam period, without annealing a radiation induced leakage current increase was observed with a rate of $\alpha \approx 8 \cdot 10^{-17} \mathrm{~A} / \mathrm{cm}$. The cluster parameters of the measured signals at different beam incidence angles could be described by empirical functions derived from earlier measurements. Finally, the APV25 multi-peak mode has been utilized to measure a system timing precision of $2 \mathrm{~ns}$ RMS and to visualize the $20 \mathrm{~ns}$ structure of the beam with the APV25 still clocked at 25 ns.

\section{Acknowledgements}

We want to express our thanks to K. Gabathuler and to D. Renker for their help with the beamline at the Paul Scherrer Institute. Moreover, we are grateful to R. Wedenig for the wire-bonding of the silicon detector module.

\section{References}

[1] CMS Collaboration, CMS Tracker Technical Design Report, CERN/LHCC 98-6, April 1998 (http://cmsdoc.cern.ch/cms/TDR/TRACKER/tracker.html)

[2] CMS Collaboration, Addendum to the CMS Tracker TDR, CERN/LHCC 2000-016, February 2000 (http://cmsdoc.cern.ch/cms/TDR/TRACKER/tracker_addendum.pdf)

[3] L.L. Jones et al., The APV25 Deep Submicron Chip for CMS Detectors, CERN/LHCC 99-09, p.162-166, September 1999 (http://www.te.rl.ac.uk/med/apv25_web/leb99_paper.pdf)

[4] M. Raymond et al., The APV6 Readout Chip for CMS Microstrip Detectors, CERN/LHCC 97-60, September 1997 (http: //www.te.rl.ac.uk/med/apv6/papers/leb_apv6_london97_paper.pdf)

[5] S. Gadomski et al., The Deconvolution Method of Fast Pulse Shaping at Hadron Colliders, Nuclear Instruments and Methods in Physics Research A 320 (1992), 217-227

[6] M. Friedl, The CMS Silicon Strip Tracker and its Electronic Readout, PhD Thesis, Vienna University of Technology, May 2001 (http: / / cern. ch/friedl $\rightarrow$ Dissertation)

[7] F. Vasey et al., Optical links for the CMS Tracker, CERN-LHCC 99-033, October 1999

(http://cern.ch/cms-tk-opto/wdocs/leb99.pdf)

[8] T. Bauer, F. Vasey, A model for the CMS tracker analog optical link, CMS NOTE 2000/056

(http://cern.ch/cms-tk-opto/perf_pubs.html)

[9] Rose Collaboration (RD48), Third RD48 Status Report, CERN/LHCC 2000-009, December 1999 (http://cern.ch/RD48/status-reports/RD48-3rd-status-report.pdf)

[10] W. Adam et al., Response of a silicon detector to protons and pions with momenta of 270, 310 and 405 MeV/c, Nuclear Instruments and Methods in Physics Research A 441 (2000), 427-437 


\section{Tables and Figures}

\begin{tabular}{|l|c|c|c|c|}
\hline \multirow{2}{*}{ Mode } & \multicolumn{2}{|c|}{ APV6 } & \multicolumn{2}{c|}{ APV25 } \\
& ENC [e] & SNR & ENC [e] & SNR \\
\hline \hline Peak & 1400 & 16 & 900 & 25 \\
\hline Deconvolution & 2250 & 10 & 1300 & 17 \\
\hline
\end{tabular}

Table 1: Typical noise (ENC) and most probable signal-to-noise (SNR) values of non-irradiated, full-size CMS detector modules measured with $350 \mathrm{MeV} / \mathrm{c}$ pions.

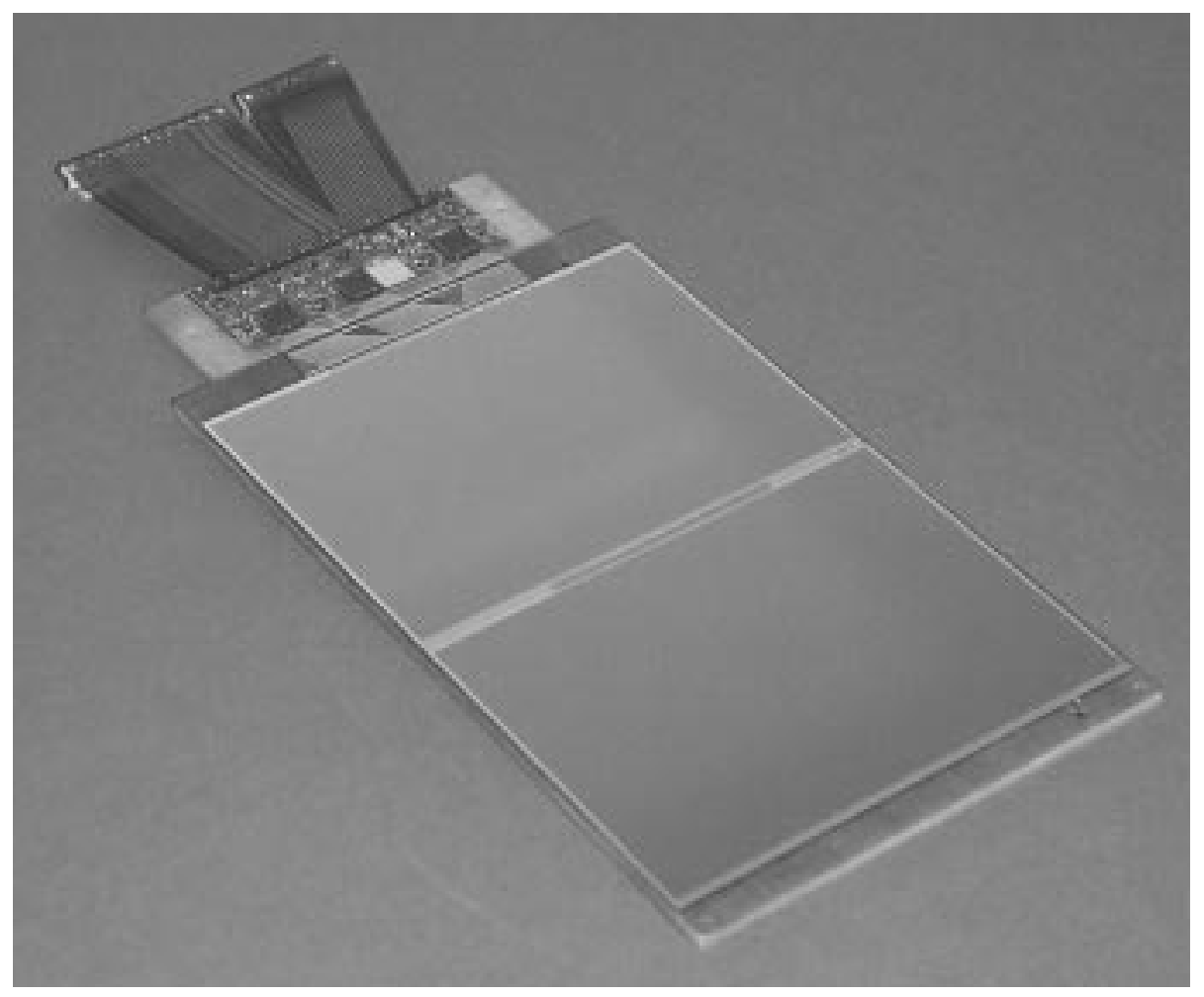

Figure 1: The APV25 silicon strip detector module. 


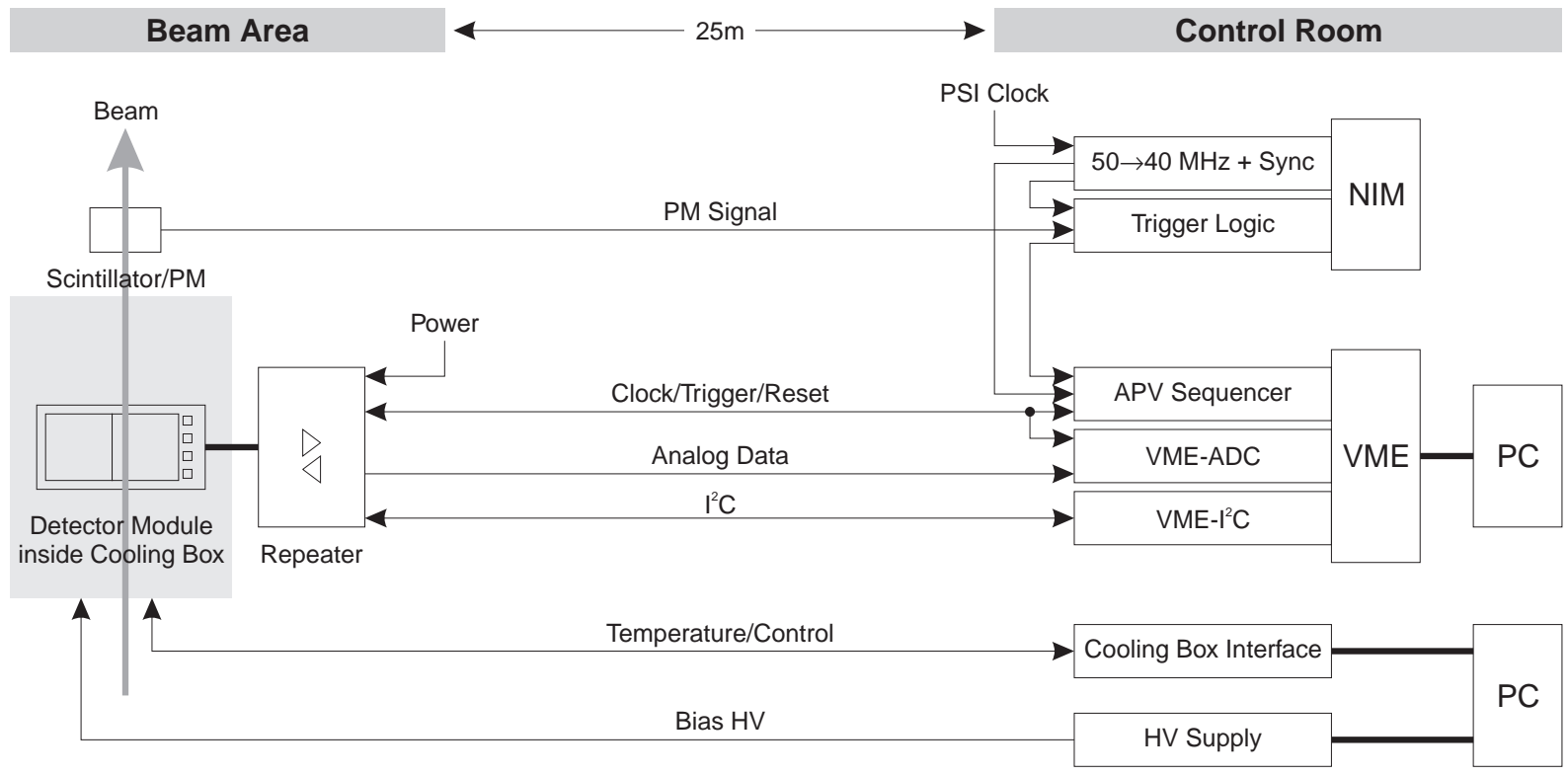

Figure 2: Block diagram of the Vienna APV setup.

Pions

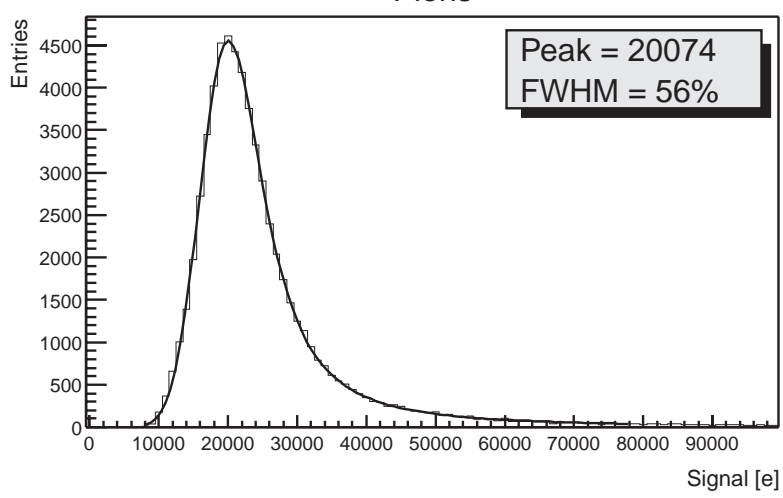

Protons

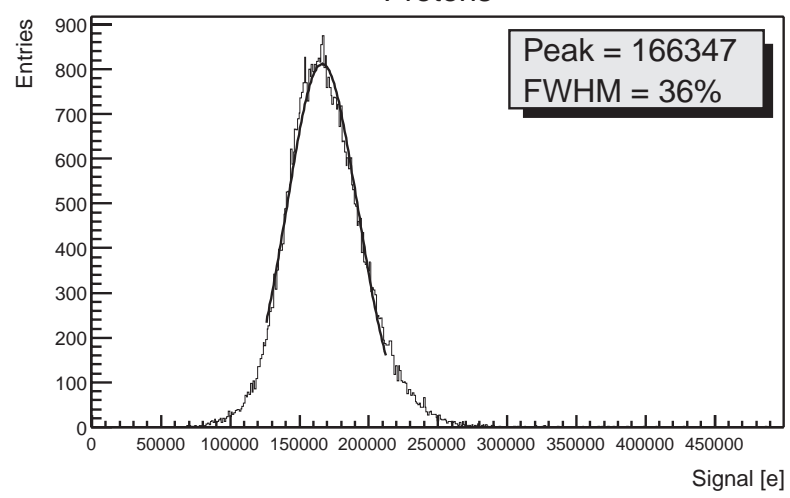

Figure 3: Typical pion (left) and proton (right) signal distributions measured at a momentum of $350 \mathrm{MeV} / \mathrm{c}$ with the APV25 chips in deconvolution mode. In the proton picture, the $\mathrm{x}$ axis range is five times larger than in the pion plot. The absolute charge scale has been obtained from internal calibration, which is accurate to about $\pm 20 \%$. 
Detector bias scan

Standard settings, $350 \mathrm{MeV} / \mathrm{c}$ pions

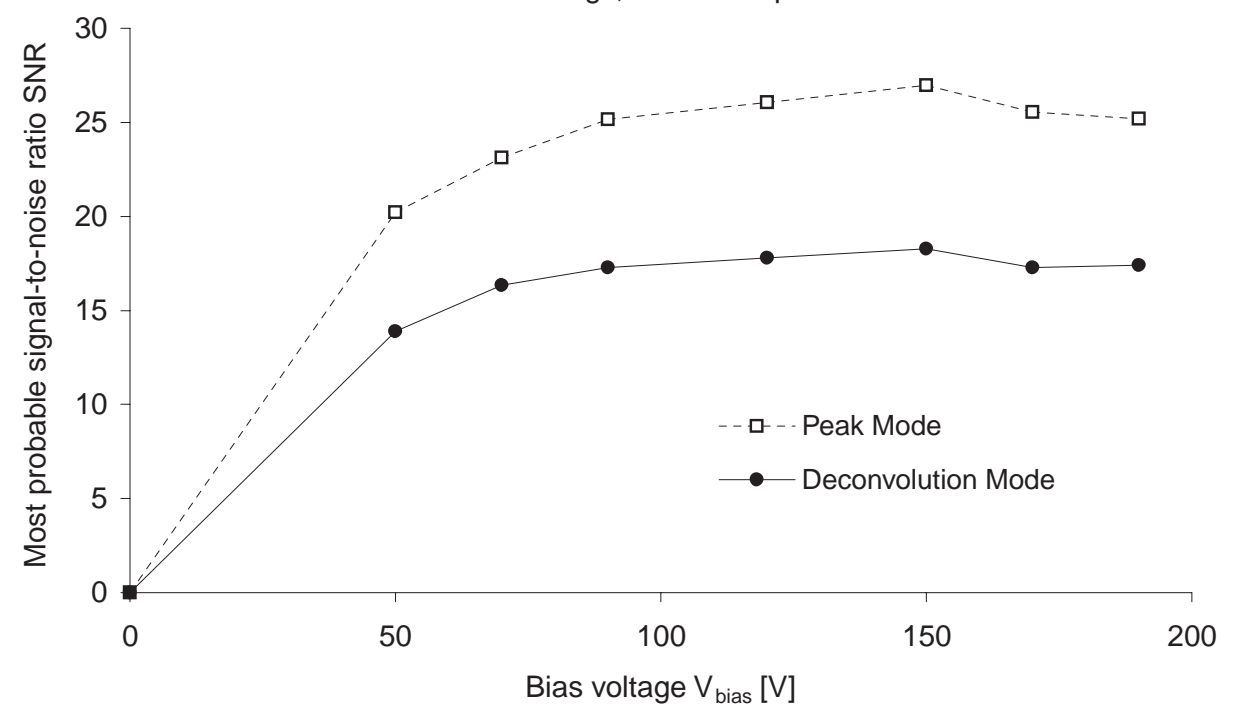

Figure 4: Signal-to-noise ratio of the APV25 silicon detector module as a function of the detector bias for $350 \mathrm{MeV} / \mathrm{c}$ pions.

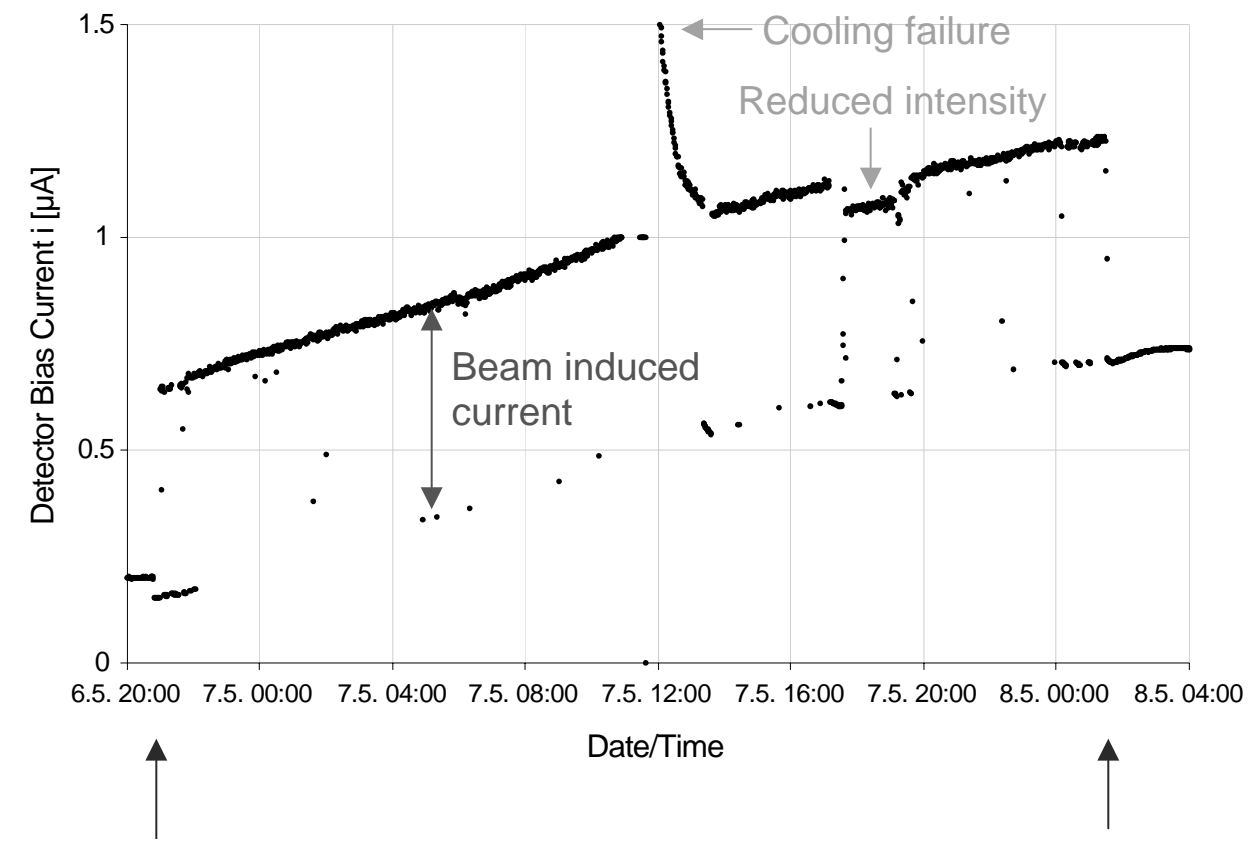

High intensity on

High intensity off

Figure 5: Detector current of the APV25 module during the high intensity period. See body text for a detailed description. 


\section{Cluster width}

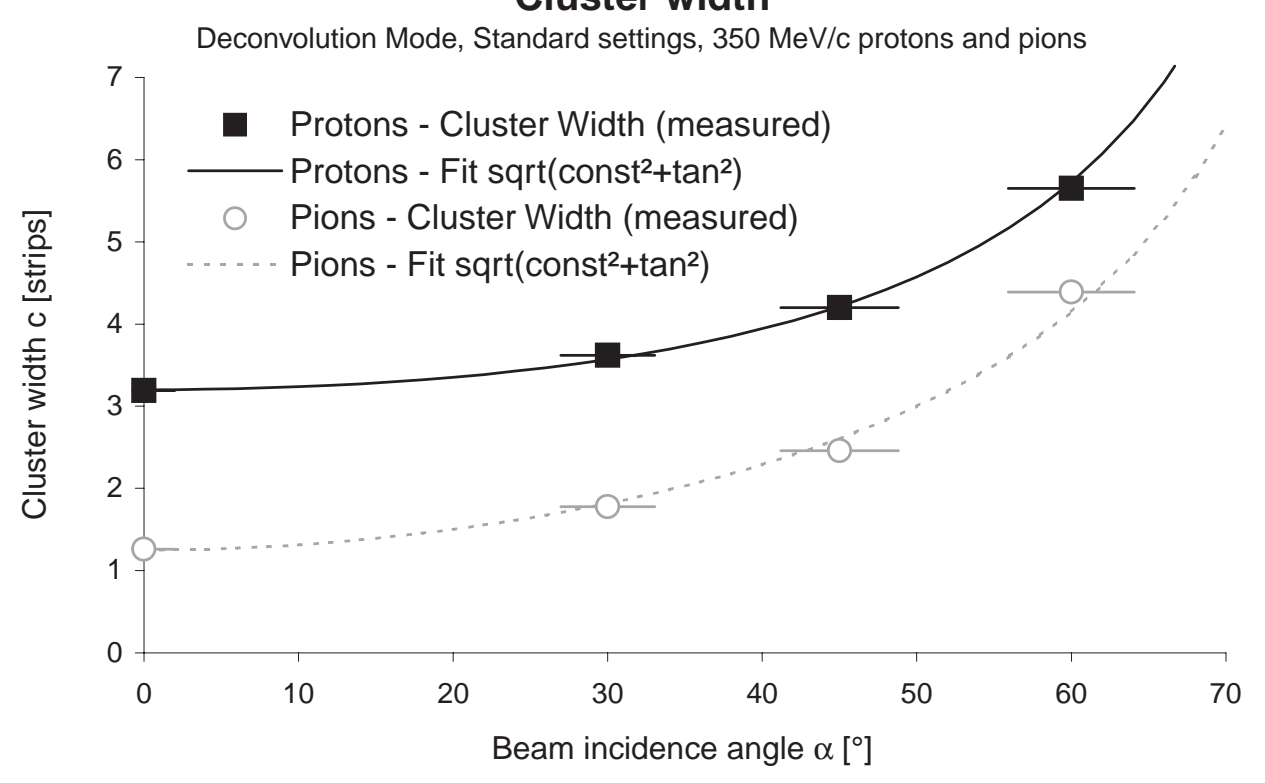

\section{Cluster signal}

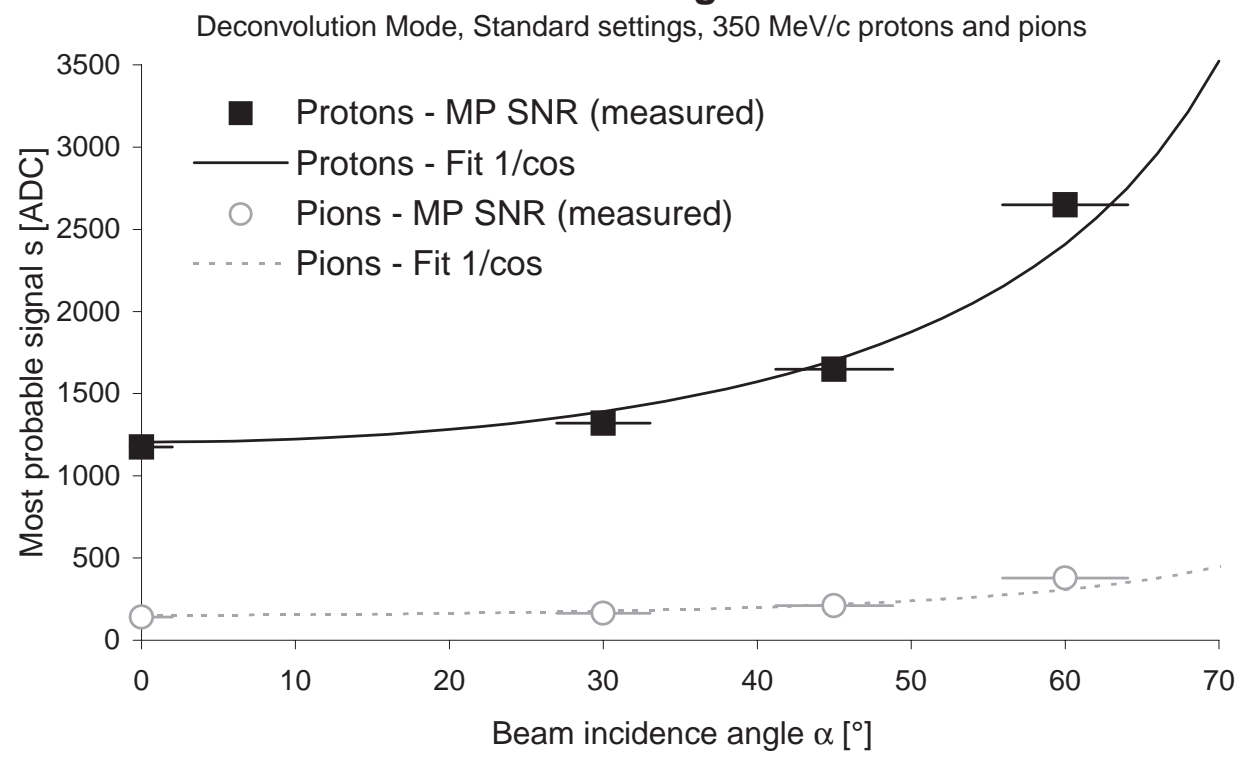

Figure 6: Angle scan of the APV25 module. Both cluster width (top) and signal (bottom) increase with the beam incidence angle. 


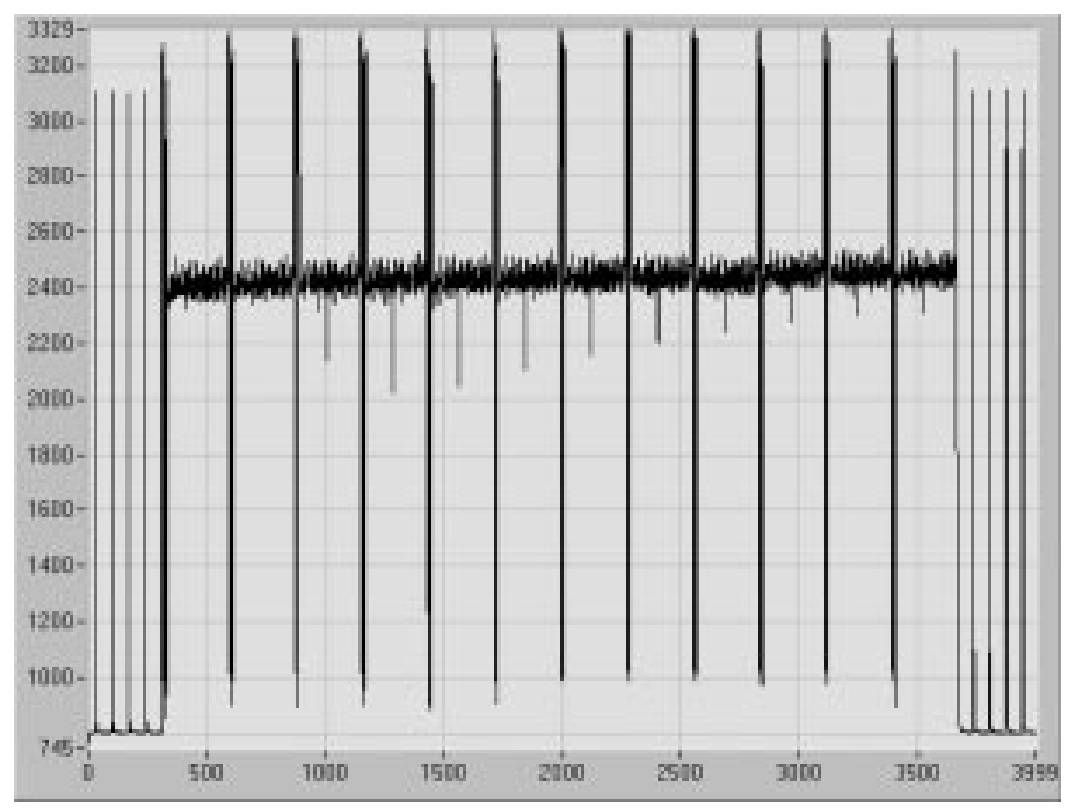

Figure 7: Screenshot of the raw APV25 output with consecutive samples obtained with a trigger sequence in multi-peak mode, representing a single particle hit. The large spikes are header data preceding each data block.

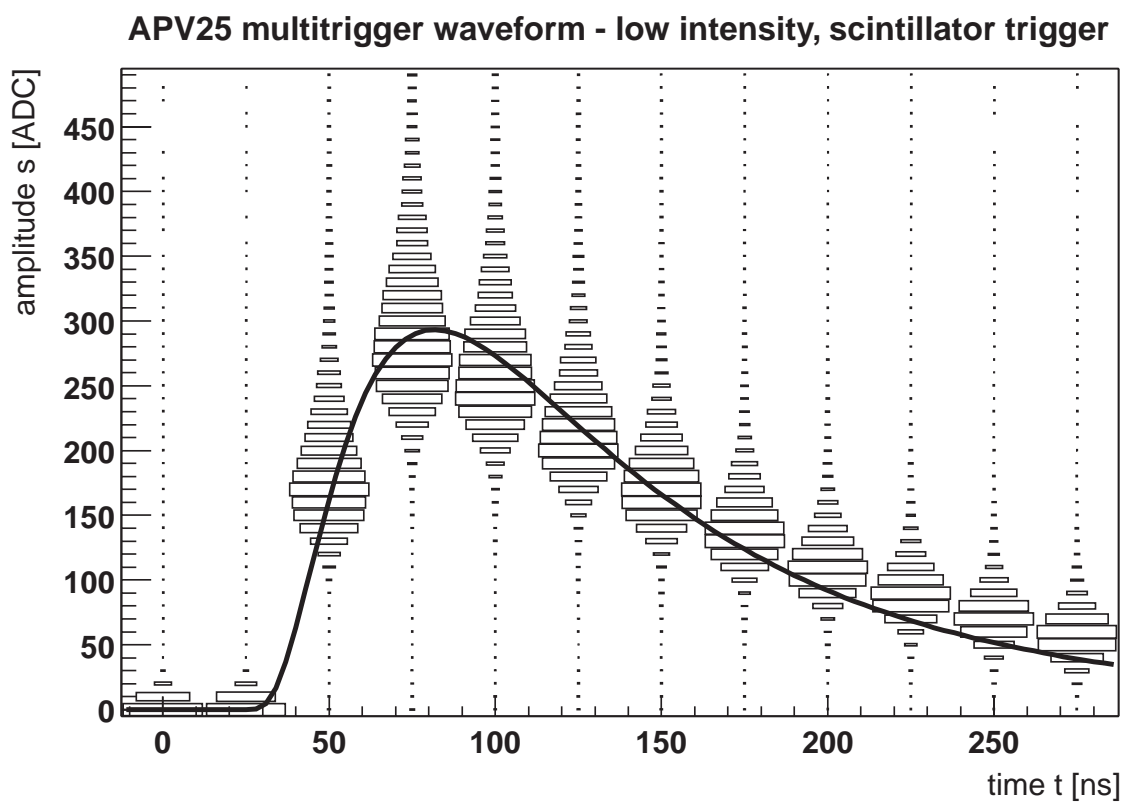

Figure 8: Overlay of the APV25 peak mode pulse shape obtained with internal calibration and the particle signal distributions spaced by the clock period of $25 \mathrm{~ns}$. The lego plots at each time slot represent Landau-like signal distributions. 


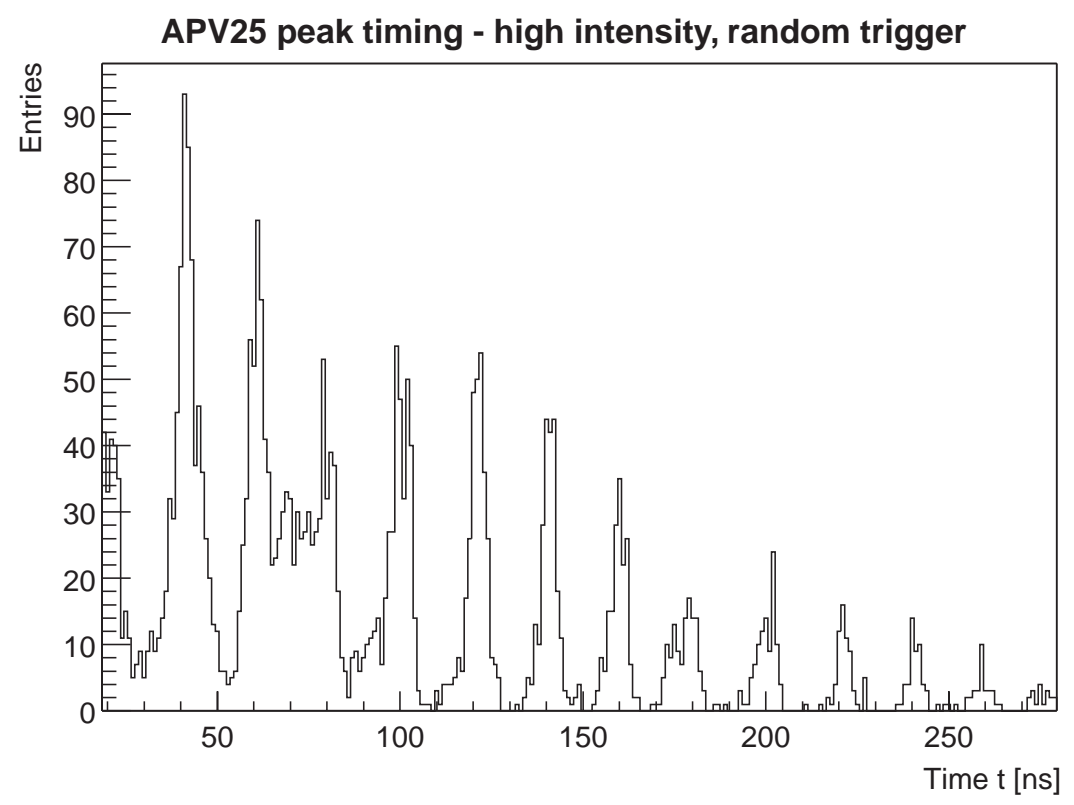

Figure 9: APV25 peaking time distribution with random trigger at high intensity, revealing the $20 \mathrm{~ns}$ PSI beam period. 